\title{
GESTIÓN DE LA AUTOMATIZACIÓN DE PLANTAS INDUSTRIALES EN CHILE ${ }^{1}$
}

\author{
José Vilaboa B. ${ }^{2}$ \\ Recibido el 11 de noviembre de 2003, aceptado el 13 de abril de 2004
}

\begin{abstract}
RESUMEN
En los últimos años, las plantas industriales chilenas vienen automatizando sus procesos de producción, lo que ellas justifican, principalmente, en el alza sostenida de los salarios industriales. Sin embargo, tanto o más importante es la puesta en el mercado de tecnologías de reciente desarrollo y bajo costo, las que viabilizan muchos proyectos que hasta hace algunos años no eran factibles. En este trabajo se analizan los aspectos económicos y de gestión, relacionados con la automatización de los procesos productivos en Chile. Como estudio de caso, se muestra la robotización de los procesos de inspección visual de la producción, lo que permite apreciar, de un modo aproximado, los beneficios y los costos económicos asociados a estos proyectos. La principal conclusión del trabajo señala que, la automatización de los procesos productivos, constituye un desafío ineludible para la industria nacional, en los próximos años.
\end{abstract}

Palabras claves: Automatización, gestión, industria chilena.

\begin{abstract}
In recent years, Chilean industrial plants have been introducing automation production processes, the main reason for this decision being the raise of industrial salaries. An important effect of such policies has resulted in the materialization of many projects - that were not feasible in the past- through the use of the new low cost technologies available. In this paper, we study the economics and management problems, related to the automation activities in Chilean companies. As a case study, we chose the robotization of visual inspection process in factory output, which balances the amount of benefits and cost involved in the projects. The main conclusion of the paper, indicates that the automation of production process is an unavoidable challenge, for the national industry, in the years to come.
\end{abstract}

Keywords: Automatization, management, Chilean industry.

\section{INTRODUCCIÓN}

En la industria chilena se está dando un proceso de automatización de los procesos productivos, incipiente pero creciente. Su objetivo es mejorar la eficiencia económica, ahorrando la contratación de mano de obra, a cambio de pequeñas inversiones. De acuerdo a un análisis de la demanda, la justificación principal de este tipo de iniciativas, deriva de una sostenida alza de salarios, y en complicaciones impuestas a la contratación de los trabajadores, por la legislación laboral. Si se observa el comportamiento de la oferta, llama la atención la reciente puesta en el mercado de tecnología, que viabiliza muchos proyectos que hasta hace algunos años no eran factibles.

En este contexto, el presente trabajo analiza las actividades a desarrollar por la industria nacional, para abordar este tipo de proyectos, desde un punto de vista económico y de gestión. Los principales antecedentes, que lo soportan, derivan de investigaciones y consultorías en que ha participado el autor.

Este tipo de soluciones integra elementos de electrónica, de computación y de técnicas matemáticas de análisis, además de componentes relacionados con la tecnología que es propia del giro de los usuarios. Para que este tipo de iniciativas evolucione positivamente, resulta necesario conseguir una adecuada complementariedad para las diversas disciplinas, lo que plantea importantes desafíos de gestión, a los jefes de proyecto.

Para estudiar el aporte de la electrónica, a la automatización de los procesos, se recomienda revisar el libro de Creus [3] y los trabajos de Jaeger [9], [10], [11], [12]. La construcción de software complejo es explicado por Fairley [4]. Los modernos desarrollos de la investigación de operaciones son descritos por Hillier y Lieberman [8].

\footnotetext{
1 Trabajo realizado en el Departamento de Ingeniería Industrial de la Universidad de Concepción.

2 Universidad de Concepción, Departamento de Ingeniería Industrial, Correo 160-C, Concepción-Chile. Fono (56) (41) 204151, jvilaboa@udec.cl
} 
Los proyectos de automatización de procesos, representan innovaciones tecnológicas importantes, con un serio impacto en la gestión de las empresas. En este contexto, los libros de Albala [1], de Ferré [5] y de Ruiz y Mandado [15], explican los fundamentos de la gestión de la tecnología.

El presente trabajo, comienza analizando la justificación de este tipo de proyectos, desde el punto de vista del desarrollo económico. Luego, se revisan las tecnologías disponibles para la automatización de procesos, y su aplicación al caso específico de la robotización de la inspección visual de la producción. Para este tipo de soluciones, se analizan los beneficios y costos asociados, y las formas alternativas de gestión de los proyectos. Al final se entregan varias conclusiones.

\section{RAZONES ECONÓMICAS PARA AUTOMATIZAR}

En esta sección se demostrará la necesidad de automatizar los procesos de producción, a medida que los países crecen económicamente. Para este propósito se recurrirá al modelo de Solow, explicado en el libro de Chiang [2]:

$\mathrm{Q}$ es la producción del país, medido como el producto geográfico bruto (PGB). C es el stock de capital, medido como la acumulación neta de inversiones, o sea, libre de depreciaciones. $\mathrm{T}$ es la fuerza laboral, medida como una fracción fija de la población.

Se supone que las materias primas son abundantes y que el crecimiento económico está limitado por las dotaciones de capital y trabajo, únicamente. Entonces, se tiene la siguiente función de producción agregada, para Q:

$$
Q=f(C, T)
$$

Se supone que la ecuación (1) tiene la forma de una función del tipo Cobb-Douglas:

$$
\mathrm{Q}=\mathrm{AC} \mathrm{C}^{\alpha}
$$

A es una constante de proporcionalidad, en tanto que $\alpha$ y $\beta$ son elasticidades. Se supone que:

$$
0<\alpha+\beta<1
$$

El nivel de automatización, se define como $\mathrm{H}$, $\mathrm{y}$ corresponde a la razón entre capital y trabajo:

$$
\mathrm{H}=\mathrm{C} / \mathrm{T}
$$

Un nivel de automatización bajo, supone la existencia de tecnología sencilla, de tipo artesanal. Un nivel de automatización alto, en tanto, indica la existencia de una tecnología sofisticada, de tipo computarizada.

A medida que pasa el tiempo (t), la fuerza laboral crece como una fracción fija de la población:

$$
\mathrm{T}=\mathrm{Be}^{\mathrm{at}}
$$

B es una constante de proporcionalidad, $t$ es el tiempo medido en años y a es una tasa de crecimiento continua.

El capital crece, a medida que pasa el tiempo, porque parte del PGB se destina a inversiones. Esto es:

$$
\mathrm{dC} / \mathrm{dT}=\mathrm{kQ}
$$

Se puede plantear la siguiente ecuación diferencial:

$$
\mathrm{dC} / \mathrm{dT}=\mathrm{kAC}^{\alpha}\left(\mathrm{Be}^{\mathrm{at}}\right)^{\beta}
$$

Donde la solución es del tipo:

$$
\mathrm{C}=\mathrm{C}(\mathrm{t})
$$

Puede demostrarse que esta economía crece a una tasa estable, pero que va bajando con el tiempo. Esto es:

$$
\mathrm{Q}=\mathrm{Fe}^{\mathrm{bt}}
$$

Donde $\mathrm{F}$ es una constante de proporcionalidad y $\mathrm{b}$ es una tasa continua de crecimiento. b baja con el tiempo. Este modelo supone que no existe innovación tecnológica.

Para lograr que la tasa de crecimiento de la economía no baje con el tiempo, se debe incentivar el desarrollo tecnológico. Matemáticamente, esto lleva a modificar el modelo, por uno más complejo.

Debe considerarse que, si se desea lograr que la economía crezca a una tasa alta, se debe ahorrar una parte importante del PGB, porque la fuerza laboral seguirá creciendo a la misma tasa histórica. Si la fuerza laboral crece en Chile $2 \%$ cada año y la economía debe crecer al $7 \%$ anualmente, entonces debe haber un esfuerzo en inversiones considerable. Este valor es actualmente cercano al $20 \%$ del PGB.

Diferenciando por t la ecuación (2), luego de haber extraído logaritmo, se tiene:

$$
\mathrm{b}=\alpha \mathrm{m}+\beta \mathrm{a}
$$


m es la tasa de crecimiento del capital. Como referencia, se estiman los valores siguientes:

$$
\begin{aligned}
& \alpha=\beta=0,45 \\
& b=7 \% \\
& a=2 \%
\end{aligned}
$$

Entonces el capital debe crecer a una tasa anual de:

$$
\mathrm{m}=13,6 \%
$$

Diferenciando por t la ecuación (4), luego de haber extraído logaritmo, se tiene que la tasa de crecimiento del nivel de automatización del país es:

$$
\mathrm{dH} / \mathrm{dt}(1 / \mathrm{H})=\mathrm{m}-\mathrm{a}
$$

Lo que da un valor de $11,6 \%$ para el crecimiento anual en el nivel de automatización del país, si se desea cumplir la meta de crecimiento de $7 \%$ anual. Este valor es sólo referencial, y tiene el propósito de ilustrar el tamaño del esfuerzo que se debe realizar.

Interpretando los desarrollos matemáticos anteriores, puede señalarse:

El crecimiento económico de los países genera un círculo virtuoso para su desarrollo, lo que obliga a la automatización de los procesos industriales: El crecimiento de la producción crea una mayor demanda de mano de obra. Al escasear ésta, se debe aumentar la productividad del trabajo en los procesos de fabricación, a través de implantar tecnologías que hagan un uso más intensivo del capital. Esto se logra desarrollando tecnologías de un mayor nivel de automatización, las que deben ser operadas por trabajadores de un mayor nivel de calificación técnica. A medida que esta situación aumenta el nivel de preparación de los trabajadores, se justifica el pago de mayores salarios. Estos mayores sueldos expanden el consumo, de modo que resulta necesario aumentar la producción.

Existe una valoración negativa, de parte de la comunidad, para la realización de innovaciones tecnológicas que provoquen cesantía, de modo que se podrían plantear reservas de este tipo para la implantación de las proposiciones. Sin embargo, y considerando las explicaciones de los párrafos anteriores, los trabajadores se ven perjudicados sólo en forma inmediata con estos proyectos, porque en el largo plazo ellos mejoran sus rentas en ocupaciones alternativas.

\section{TECNOLOGÍAS PARA LA AUTOMATIZACIÓN DE PROCESOS}

En las últimas décadas se viene dando un fuerte desarrollo de tecnologías del ámbito de la computación, de la electrónica y de métodos matemáticos de análisis. A través de una aplicación integrada de las mismas, se facilita el desarrollo de aplicaciones innovadoras, para la automatización de los procesos de producción. Estas nuevas tecnologías complementan a las tradicionales y permiten bajar sustancialmente las inversiones en juego. De este modo viabilizan muchas soluciones que no resultaban posibles hace algunos años.

En resumen, las tecnologías disponibles para el control de los procesos de producción, son:

\section{a) Sistema manual}

Se instalan instrumentos, los que miden las variables que identifican el estado del proceso productivo, y dan la información a través de la lectura en visores. Un operario lee los instrumentos y decide corregir el proceso. Para este fin, mueve accionamientos mecánicos, usando su cuerpo. A modo de ejemplo, puede cerrar una válvula a través del movimiento de giro de una palanca.

\section{b) Sistema mecánico}

Usando el concepto de retroalimentación, se puede diseñar y construir un sistema mecánico, el que actúa sobre los accionamientos, según lo que le señala un instrumento que mide cierta variable del proceso. Como ejemplo de este tipo de soluciones, está el divulgado uso de un flotador, el que abre y cierra una válvula para el llenado de una cuba, según si baja o sube con el nivel de agua en el estanque.

\section{c) Sistema electrónico simple}

Es similar al caso anterior, pero la información se transmite a través de circuitos electrónicos. El instrumento traduce a una señal eléctrica sus mediciones y un circuito analiza la señal, para activar o desactivar un accionamiento eléctrico (generalmente un pequeño motor), el que mueve los elementos mecánicos que modifican el proceso.

\section{d) Uso de PLC (dispositivo lógico programable)}

En este caso, las señales eléctricas emitidas por un grupo de instrumentos, son analizadas por un procesador de bajo rendimiento, el que, a través de una adecuada programación, emite señales para activar o desactivar los accionamientos eléctricos. 


\section{e) Uso de Computadores Personales}

Esta es la tecnología que se ha divulgado en los últimos años. El uso de PLC es reemplazado por computadores personales (PC). Esta tecnología permite acceder a abundante software y a hardware de bajo precio.

\section{f) Uso de Sistemas Integrados}

Recientemente, empresas líderes a nivel mundial, han puesto en el mercado un completo equipamiento de hardware y sus interfaces electrónicas, las que son muy fáciles de integrar en las soluciones. Esto se complementa con la venta de software que permite programar las soluciones, con un gran ahorro de tiempo.

Un punto clave, en el desarrollo de estas aplicaciones, es que ellas requieren del uso de sistemas operativos que trabajen en tiempo real. Esto anula la posibilidad de usar WINDOWS de Microsoft, en la mayoría de las aplicaciones complejas, de modo que no se puede recurrir al abundante software que opera bajo esta modalidad, tales como EXCEL, ACCESS, aplicaciones de VISUAL BASIC, etc. Esto significa que se debe recurrir a las ya tradicionales (pero obsoletas) soluciones que operan bajo DOS, construyendo los programas de computación con lenguajes tales como $\mathrm{C}++$.

Pareciera que lo anterior ha sido un descuido de Microsoft, el líder mundial indiscutido en desarrollo de software para PC. Esto ha abierto oportunidades para la creación de competencia en esta área, para empresas que operan en el ámbito de la electrónica. Estas empresas ofrecen abundante software para programar soluciones relacionadas con la automatización de procesos productivos, las que deben hacer uso de sistemas operativos aportados por ellos mismos. De este modo, pueden plantear importantes amenazas en el futuro al software que opera bajo WINDOWS.

Así, en el caso de NATIONAL INSTRUMENTS, ellos ofrecen hardware y software para construir sistemas para la automatización de procesos, los que permiten cubrir 5 niveles del problema [14]:

\section{a) Primer Nivel}

Como elementos que entran en contacto directo con el sistema productivo, existen tres grupos: Sensores que cubren los requerimientos tradicionales, tales como medidores de temperatura, de presión, etc., los que permiten medir el estado de los procesos de producción. Cámaras Digitales que permiten acceder a modernas soluciones basadas en el procesamiento de imágenes. Motores y otros accionamientos eléctricos, con los que se puede actuar modificando el proceso de producción.

\section{b) Segundo Nivel}

Se proporciona equipamiento para conectar a los elementos anteriores, con los PC. Para este fin, se ofrecen acondicionadores de señales, relés y otros elementos para la transmisión de datos, tales como diversas tarjetas para puertas seriales, paralelas, USB, etc.

\section{c) Tercer Nivel}

Para uso industrial, y soportando condiciones de operación rigurosas, se ofrecen computadores, que tienen las mismas configuraciones que los tradicionales PC de oficina. Sin embargo, se diferencian en soportar agresiones del tipo: presencia de polvo y humedad, vibraciones, campos eléctricos y magnéticos, ondas electromagnéticas, diferencias de temperatura, etc.

\section{d) Cuarto Nivel}

Estos PC pueden ser conectados en red, y de este modo pueden alimentar a bases de datos, donde se registra el avance en el proceso de producción, las que suelen estar soportadas en computadores centrales de alto rendimiento. Esta información se integra a los demás sistemas de información para la operación de la empresa.

\section{e) Quinto Nivel}

Se dan facilidades para acceder a todos los elementos de estas configuraciones, a través de INTERNET. De este modo, un ejecutivo del área de producción, puede estar de viaje en un lugar lejano al de ubicación de la fábrica, y puede modificar las condiciones de operación del proceso productivo, con el nivel de detalle que desee. Por ejemplo, cierta válvula deberá cerrarse cuando un medidor de temperatura señale 300 Grados Celsius, modificando el anterior índice de 280.

El problema, en el uso de esta tecnología de tipo integrada, radica en que las soluciones obligan a incurrir en elevados costos. Esto pasa por la compra de un abundante software para la programación de las soluciones, lo que "amarra" la compra de los elementos de hardware. El principal beneficio, de recurrir a estos proveedores, radica en importantes ahorros en el trabajo de los ingenieros que aborden los proyectos de automatización.

Alternativamente, se puede recurrir a soluciones de menor costo, pero que exigen la realización de mucho trabajo de ingeniería. Para este fin, se deberá programar software que opere bajo DOS, se deberá encargar la fabricación de PC a armadores nacionales usando el concepto de "clones" y se deberá recurrir a INTERNET para proveerse de los acondicionadores de señales, de los 
relés y otros elementos electrónicos para las interfases. Comparativamente, este tipo de soluciones deberá ser priorizada en países donde el trabajo de ingeniería es de bajo costo, como es el caso de Chile.

\section{EL CASO DE LA AUTOMATIZACIÓN DE LOS PROCESOS DE INSPECCIÓN VISUAL DE LA PRODUCCIÓN}

Los procesos de inspección visual de la producción, constituyen actividades industriales de gran importancia en Chile. Actualmente, lo usual es que este trabajo sea realizado manualmente por obreras. Sin embargo, desde que están disponibles las nuevas tecnologías, descritas en la sección anterior, resulta posible robotizar este tipo de trabajos. Este último tipo de soluciones ofrece grandes beneficios económicos, por concepto de ahorros en salarios, entre otras utilidades, los que suman utilidades muy superiores a las inversiones involucradas.

Según los especialistas en desarrollo económico, Chile avanza desde una etapa exportadora primaria, en que se venden materias primas con un bajo valor agregado, hacia una fase exportadora secundaria, en que se aumenta el valor agregado de la producción. Esto último exige la creación de industrias y el desarrollo de nuevas tecnologías, para la creación de nuevos productos y de nuevos procesos.

En ambas situaciones, las empresas que reciben las materias primas desde quienes las extraen del medio, deben realizar un trabajo de inspección visual. De este modo se las puede clasificar según las diversas variables que señalan sus atributos. Una característica inherente a los bienes que genera la naturaleza directamente, radica en una amplia variabilidad de sus características. En general, esta variabilidad es mucho menor cuando se trata de productos creados por el hombre, a través de procesos industriales. A modo de ejemplo, el zumo de manzana envasado en una fábrica, es mucho más homogéneo que las manzanas recolectadas en un predio agrícola.

Por la razón anterior, en el futuro en Chile, aumentará la importancia de estos procesos de inspección visual, más allá de la que ya tienen hoy. Esta situación obliga a las empresas chilenas que exportan materias primas, ya sea con poco o mucho valor agregado, a estudiar mejoras tecnológicas para esta importante fase de la producción.

De gran importancia, para el desarrollo de este tipo de proyectos, resulta la puesta en el mercado, en la última década, de cámaras de televisión de bajo costo, las que hacen uso de tecnología digital. Ellas facilitan enormemente el desarrollo de sistemas para el procesamiento de imágenes y están desplazando a otras tecnologías basadas en scanners, ultrasonido, rayos $\mathrm{X}$, etc. La teoría del tratamiento digital de imágenes se da en los libros [6] y [7]. El documento [13] muestra equipo y software, para el procesamiento de imágenes, de desarrollo reciente.

El autor ha realizado varias investigaciones y consultorías, con las que ha podido acotar la tecnología que se debe emplear. Un ejemplo de este tipo de aplicaciones se muestra en el artículo [17], donde se describe la construcción de una máquina prototipo para la automatización de la selección de la fruta en los packing. Otro ejemplo se da en el artículo [16], el que muestra varias oportunidades para un mejor aprovechamiento de la materia prima en los procesos de aserrío de rollizos.

En resumen, este tipo de tecnología captura las imágenes con cámaras de TV y las lleva a la memoria de un computador. De este modo, un software las puede analizar modificando el proceso productivo, a través de activar determinadas interfases electrónicas.

Una de las aplicaciones más simples, radica en la construcción de sistemas para la selección de la producción, lo que exige integrar las siguientes partes:

ALIMENTACION. La producción debe ser ubicada ordenadamente y con una determinada frecuencia sobre el inicio de la mesa de filmación. La solución a este problema exige la construcción de dispositivos mecánicos que son específicos al tipo de producto.

MESA DE FILMACION. La materia prima debe desplazarse por la mesa de filmación para que la cámara de TV pueda filmarla. Este problema requiere de soluciones que deben ser proporcionadas por especialistas en el área tecnológica a que corresponde el giro de cada cliente. Para que el sistema pueda analizar todas las caras de los productos, se puede dar un movimiento de rotación a ellos, o bien, se les puede filmar simultáneamente con varias cámaras.

CAMARA DE TV. Se emplean cámaras de TV digitales (monocromáticas o en colores, según los requerimientos de la aplicación), las que filman la producción que se mueve sobre la mesa y envían la señal a la computadora. Estos elementos requieren de una adecuada protección contra la humedad, el polvo, los campos y ondas electromagnéticas, las vibraciones, etc. y de un adecuado sistema de iluminación. La cantidad de cámaras a usar es proporcional al volumen de producción por unidad de tiempo a procesar y al tamaño de los detalles a detectar.

COMPUTADORA. Con las modernas computadoras personales (PC's) resulta posible resolver la mayoría de los problemas. Para algunas soluciones se deben integrar 
varios PC's que comparten el procesamiento de la información. Eventualmente, puede ser necesaria la construcción de un hardware basado en multiprocesamiento.

SELECTOR. Una vez que la computadora ha analizado las imágenes y ha decidido que determinada unidad de producción debe ser aceptada o debe ir a rechazo, se activa automáticamente una interfase que lleva a un selector a la posición correcta. Se debe construir un dispositivo mecánico sencillo que traslade al rechazo y a la producción aceptada a los lugares que señale el usuario.

\section{BENEFICIOS DE LAS SOLUCIONES}

El principal beneficio, derivado del uso de estos sistemas, radica en importantes ahorros en mano de obra directa (MOD), porque los sistemas reemplazan el trabajo manual de los operarios.

A modo de ejemplo, con el uso de esta tecnología, un packing típico reemplaza a 150 trabajadoras, las que realizan directamente el proceso manual de selección. Si se estima el salario mensual en 180.000 pesos, se tienen ahorros anuales de 324 millones de pesos, sólo por un menor uso de MOD.

Estimativamente, esta solución requiere de una inversión inicial de 100 millones de pesos, de modo que sólo con los ahorros por MOD, se puede recuperar la inversión en cuatro meses.

Otros beneficios cuantificables en dinero, son:

- Ahorros en personal indirecto y administrativo, porque se podrá prescindir del servicio de algunos supervisores, de encargados de bienestar, de funcionarios para selección de personal, etc.

- $\quad$ Ahorros en infraestructura destinada a dar servicios al personal (casino, baños y duchas, posta, etc.), las que podrán ser de menor tamaño en el futuro.

- Ahorros en servicios dados por contratistas, tales como servicios de locomoción, servicios de colación, etc.

- Disminuye el deterioro de los productos durante la manipulación, lo que frecuentemente constituye un alto porcentaje de pérdidas, con lo que existe un mejor aprovechamiento.
En forma adicional a los beneficios que pueden ser estimados en dinero, existen otros que son difíciles de cuantificar:

- Se mejora la posición corporativa en el largo plazo, en un escenario con salarios en alza y de escasez de mano de obra.

- Se mejora la capacidad de negociación con los sindicatos.

- Disminuye la incertidumbre en el trabajo de control de calidad, porque las máquinas tienen un comportamiento homogéneo.

- Mejoran los resultados del trabajo de selección, porque las máquinas cometen menos errores.

- Estos sistemas permiten llevar registros históricos del proceso de selección lo que, junto al uso de software estadístico, permite mejorar la toma de decisiones en el ámbito de la administración superior.

Otros beneficios derivan de las ventajas técnicos que ofrece el procesamiento computacional de imágenes:

- Se pueden emitir informes resumen, con los resultados del análisis de las imágenes.

- $\quad$ Se pueden crear bases de datos para llevar registros estadísticos con las conclusiones del análisis de las imágenes.

- Se pueden guardar imágenes en disco duro, con importantes ahorros monetarios respecto del uso de videograbadoras.

- Se producen importantes ahorros de tiempo del personal, al no tener que revisar imágenes que no aportan información. Este trabajo automático de selección de imágenes grabadas no lo pueden realizar las videograbadoras.

\section{COSTOS DE LAS SOLUCIONES}

Los costos de estas soluciones radican en la compra de los elementos físicos y de software y en el pago de los servicios de instalación y mantención (a esto último se le denomina soporte). Existen diversos proveedores para estos elementos, con marcadas diferencias en precios, calidades, servicio post-venta y adaptabilidad de los sistemas. 
Para dar una referencia de los valores en juego, se resume una cotización realizada por un aserradero ubicado en la VIII Región. Se consultó los precios de scanners para medir el volumen de madera aportado por cada rollizo, entre varios proveedores. Estos incluyen el hardware, el software y los implementos electrónicos y mecánicos. Los sistemas nacionales incluyen el soporte técnico, en tanto que los importados lo cobran separadamente:

- Sistema de origen alemán (año 1995) muy completo, sin soporte \$ 800.000.000.

- Sistema de origen canadiense (año 1997) más simple, sin soporte $\$ 95.000 .000$

- Sistema de origen nacional (año 1997) muy sencillo, con soporte $\$ 25.000 .000$

- $\quad$ Sistema construido a pedido por una empresa de ingeniería de la VIII Región (año 1998) similar al canadiense, con soporte $\$ 12.000 .000$

Una de las variables críticas, que debe ser estudiada cuando se incorporan estas soluciones, está constituida por los servicios de instalación de los sistemas. En algunos casos, la venta de los elementos físicos incluye estos servicios y en otros casos ellos son cobrados en forma separada. Se deben considerar, entre otros:

- $\quad$ el montaje de los elementos físicos

- la capacitación en el uso del sistema

- las adaptaciones a realizar a elementos físicos y software

- $\quad$ la resolución de problemas que surgen al cabo de algún tiempo (después de un año, por ejemplo)

Este tipo de servicios presenta una marcada diferencia para un aprovisionamiento nacional y uno extranjero.

Los proveedores extranjeros suelen entregar el servicio de post-venta a través de enviar a asesores por plazos a fijar por los clientes. Ellos suelen cobrar honorarios por un monto de US\$ 10.000 al mes por ingeniero. Adicionalmente, se deben cancelar los pasajes de y al país de origen, los servicios de un buen hotel y traslado hotel-empresa-hotel. Frecuentemente, la instalación de los sistemas requiere traer a uno o dos especialistas por uno o dos meses. Si el sistema falla, se deben contratar nuevamente los servicios citados, lo que es lento y caro.

Las empresas de ingeniería chilenas poseen ventajas comparativas, en la entrega de estas prestaciones, cuyo origen radica en la cercanía geográfica entre proveedores y clientes, en los menores sueldos que se pagan en Chile a los ingenieros y en el conocimiento de la mentalidad de los clientes, entre otros. Esto permite bajar los costos de los servicios de soporte, con los consiguientes ahorros para los clientes.

\section{ALTERNATIVAS EN LA DEFINICIÓN DE LOS PROYECTOS}

El desarrollo de este tipo de soluciones requiere de la integración de tecnologías de origen variado, lo que sólo se logra mediante la creación de equipos de trabajo interdisciplinarios. Esto exige relevantes condiciones profesionales y personales para los ingenieros que lideren las iniciativas. El libro [18] estudia el ciclo de los proyectos de un modo integrado y ofrece una pauta para guiar este tipo de trabajo.

Las empresas chilenas, cuentan con cuatro opciones básicas, para proveerse de los servicios de ingeniería, que estas iniciativas requieren:

\section{a) Desarrollo interno}

Se puede recurrir a un desarrollo interno, por ingenieros de la empresa. Esta sería una estrategia que avanza en la dirección contraria a la externalización de los servicios, lo que constituye una práctica generalizada en las empresas nacionales. Sólo se justificaría en la necesidad de mantener la confidencialidad de los desarrollos, por compañías de gran envergadura.

\section{b) Outsourcing nacional}

Se pueden formular proyectos a ser desarrollados por empresas de ingeniería chilenas, dando soluciones "a la medida". A tal fin es necesario que la empresa usuaria posea una contraparte técnica, que supervise el desarrollo de las iniciativas.

c) Sistemas de uso general

Se puede comprar tecnología de uso general, en el extranjero. En tal caso queda pendiente el problema de la instalación y los servicios post-venta.

\section{d) Proyectos "llave en mano"}

También se pueden desarrollar proyectos "llave en mano" con proveedores del extranjero. Sin embargo, esta opción obliga a incurrir en altos costos.

Se considera que las empresas chilenas, de tamaño mediano y pequeñas, deben descartar las opciones "a" y 
“d”. La opción "a" supone contratar de un modo permanente, a un numeroso equipo de ingenieros, que cubra las diversas especialidades involucradas en las iniciativas. La opción “d”, en tanto, obliga a incurrir en altos costos para financiar el trabajo de ingenieros extranjeros que operarán en Chile, mientras se implementan los proyectos.

En resumen, para la mayoría de las empresas nacionales, el pago de una consultoría a oferentes nacionales representa sustanciales ahorros respecto de la adquisición de sistemas de uso general que se venden en el extranjero, además de otras ventajas. Sin embargo, las empresas que contraten servicios de ingeniería nacionales, deberán asumir riesgos y deberán realizar un trabajo de gestión de los proyectos, que les significará mayores problemas, que si recurren a proveedores del extranjero.

Para sacar adelante los proyectos, está disponible en Chile, todo el equipamiento electrónico y de hardware, que se requiere en estos casos: computadoras personales de bajo precio, que son armados en el país, y elementos electrónicos para todo tipo de requerimientos, que pueden ser adquiridos a través de INTERNET. Adicionalmente, se cuenta con personal calificado para programar software matemático. Sin embargo, hay pocos profesionales y académicos, que estén en condiciones de integrar todas las componentes tecnológicas de las soluciones, de modo que aquí están los principales desafíos para que estas iniciativas prosperen.

Debe considerarse que, en Chile, los proyectos de tipo tradicional, relacionados con la creación de grandes plantas industriales, suelen ser abordados por empresas de ingeniería y proveedores de bienes de capital del extranjero. Los proyectos de nuevo tipo, en tanto, abren oportunidades que debieran ser asumidas por la ingeniería chilena.

\section{CONCLUSIONES}

Como se ha explicado, la automatización de las plantas industriales ofrece grandes oportunidades para el desarrollo de proyectos de inversión de alta rentabilidad, a las empresas chilenas. Actualmente, este proceso presenta características incipientes, y debiera generalizarse en algunos años más.

Debe considerarse que, en el contexto de una economía competitiva, las empresas están obligadas a ser eficientes. Aquellas corporaciones que no tomen las decisiones adecuadas o no se actualicen frente a los cambios que se generan en el entorno, están condenadas a perder rentabilidad y desaparecer. De este modo, la automatización de los procesos de producción constituye una necesidad ineludible, para las empresas chilenas.

La explicación anterior vale como una justificación, de tipo general, para que la industria chilena avance hacia una mayor automatización de los procesos de producción. A partir de mediados de la década de los ochenta, el país presentó un alto crecimiento económico, hasta que en el año 1998 se inicia un cierto estancamiento. Esto puede verificarse consultando las estadísticas oficiales del Estado Chileno, sobre crecimiento del Producto Geográfico Bruto (PGB). Mientras la economía creció a tasas altas, hubo una sostenida alza de salarios, lo que llevó a una pérdida de ventajas competitivas para los procesos productivos intensivos en el uso de la mano de obra. Desde 1998, muchas empresas han realizado reducciones en los salarios, lo que ha afectado, principalmente, a los ejecutivos e ingenieros $\mathrm{y}$, en menor medida, al personal de menor calificación. La evolución de los sueldos en Chile, es objeto de debates y comentarios, por los profesionales del sector productivo de la Región del Biobío, donde se ha establecido la apreciación anterior. Sin embargo, se espera que en el futuro la economía vuelva a crecer a tasas altas, con el alza de salarios asociada. Entonces, será necesario que las empresas nacionales estén preparadas para salvar los nuevos desafíos.

Con la excepción de unas pocas grandes corporaciones, las empresas chilenas presentan un deficiente manejo de la innovación tecnológica. Esta es una apreciación compartida por todos los especialistas, tanto del sector productivo como académicos. Los proyectos de automatización requieren proveerse de nuevas tecnologías y su adquisición en el extranjero tiene costos muy altos, y puede hacer no rentables, a muchas iniciativas. Alternativamente, su desarrollo en Chile exige incursionar en actividades de Investigación y Desarrollo, para las cuales las empresas no están preparadas. Esta última opción, sin embargo, permitiría acceder a ahorros sustanciales en las inversiones para construcción de equipos y software, y en los gastos posteriores a la puesta en marcha. La posibilidad de un desarrollo nacional de las tecnologías que los proyectos de automatización necesitan, resulta totalmente válida, si las empresas modifican algunas prácticas de gestión a las que están acostumbradas.

\section{REFERENCIAS}

[1] A. Albala; 'Planificación estratégica de la innovación tecnológica", Ediciones del Colegio de Ingenieros de Chile, Santiago de Chile, 1992. 
[2] A. Chiang; 'Fundamental methods of mathematical economics", McGraw-Hill, New York - USA, 1974.

[3] A. Creus; 'Instrumentación Industrial", Alfaomega, Ciudad de México, 1998.

[4] R. Fairley; 'Ingeniería de Software", McGraw Hill, Ciudad de México, 1987.

[5] R. Ferre; 'El departamento de I+D: Organización y Control", Ediciones Marcombo, Barcelona, España, 1990.

[6] R. González, P. Wintz; 'Digital image processing", Addison Wesley, New York, USA, 1977.

[7] R. González, R. Woods; "Tratamien to digital de imágenes", Addison Wesley, Wilmington, Delaware, USA, 1996.

[8] F. Hiller, G. Lieberman; 'Introducción a la Investigación de Operaciones", McGraw Hill, Ciudad de México, 1980.

[9] R. Jaeger; "Tutorial: analog data acquisition technology. Part I: Digital to analog conversion", IEEE Micro, pp. 20-37, Mayo 1982.

[10] R. Jaeger; "Tutorial: analog data acquisition technology. Part II: Analog to digital conversion", IEEE Micro, pp. 46-56, Agosto 1982.

[11] R. Jaeger; "Tutorial: analog data acquisition technology. Part III: Sample and holds, instrumentation amplifier and analog multiplexer", IEEE Micro, pp. 20-35, Noviembre 1982.

[12] R. Jaeger; 'Tutorial: analog data acquisition technology. Part IV: System design, analysis and performance", IEEE Micro, pp. 52-61, Febrero 1983.

[13] National Instruments; 'PC-Based Vision Solutions", Ediciones de National Instruments, USA, 1999.

[14] National Instruments; 'Data Acquisition and Signal Conditioning", Ediciones de National Instruments, USA, 2001.

[15] M. Ruiz, E. Mandado; 'La innovación tecnológica y su gestión", Ediciones Marcombo, Barcelona España, 1989.

[16] J. Vilaboa; 'Oportunidades para la incorporación de software que optimice el corte de rollizos en los aserraderos chilenos", Revista Facultad de Ingeniería, Universidad de Tarapacá, Arica - Chile, pp. 03-11, Diciembre 1997.

[17] J. Vilaboa; "Automatización de la selección de la fruta en los packing", Revista Facultad de
Ingeniería, Universidad de Tarapacá, Arica - Chile, pp. 03-08, Diciembre 1999.

[18] J. Vilaboa; 'Ingeniería de Proyectos: Un análisis integrado", Ediciones de la Dirección de Docencia de la Universidad de Concepción, Concepción Chile, 2001. 